\title{
Effects of mineral water from spring 3 in Băile Tuşnad on experimentally induced alcoholic liver disease
}

\author{
Gabriela Dogaru', Marieta Motricală², Molnár Ákos2, Vasile Rus3
}

1. “Iuliu Hatieganu” University of Medicine and Pharmacy Cluj-Napoca, Romania Clinical Rehabilitation Hospital Cluj-Napoca 2. Tuşnad Spa Complex - Băile Tușnad

\section{ABSTRACT}

3. University of Agricultural Sciences and Veterinary Medicine, Cluj-Napoca, Romania

Chronic alcohol consumption leads to the development of specific disorders, particularly of the liver. In this study, we aimed to investigate by optical microscopy the hepatic changes occurring after administration of ethyl alcohol for 70 days to Wistar rats, as well as the curative effect of mineral water from spring 3 in Băile Tuşnad administered for 50 days after completion of ethyl alcohol administration. Liver fragments were harvested and histologically processed by paraffin embedding and stained with Goldner's trichrome method. After the first 70 days of the experiment, in the liver parenchyma, fat loading of hepatocytes and mild perivascular fibrosis were identified. After another 50 days, during which some of the rats drank tap water, the pathological evolution did not seem to stop, but on the contrary, a slight aggravation could be observed, while in rats drinking Tuşnad mineral water there was an arrest of evolution and a slight regression of the described aspects. This study evidences the curative effect of mineral water from spring 3 in Băile Tuşnad on alcoholic liver disease.

Key words: alcoholic liver disease, Tuşnad mineral water, microscopic aspects.

\section{Introduction}

In alcoholic liver disease (ALD), liver lesions cover a wide spectrum from steatosis to cirrhosis [1]. The most typical aspects are hepatocyte ballooning, cytoplasmic rarefaction, cytoskeletal destruction, and even Mallory-Denk body formation. These changes initially occur in the centrilobular area, after which they extend towards the lobule periphery, where lesions are increasingly pronounced [2]. Hepatic steatosis and steatohepatitis are frequently found in persons who consume large amounts of ethyl alcohol. Ethyl alcohol induces hepatic steatosis by alteration of fatty acid metabolization mechanisms [3]. The defensive mechanism against the toxic action of ethyl alcohol provided by peroxisomes is more reduced in women as well as in female rats, so that in these, large amounts of free fatty acids can accumulate, which are sufficient to induce membrane lesions, causing necrosis, inflammation and progression towards fibrosis and cirrhosis [4]. The predisposition of women to severe liver diseases following alcohol consumption is also reported by other authors [5]. Fibrosis processes are present around the centrilobular venule, but they can also extend to the perilobular venule [6], and trichrome staining allows to accurately assessing the fibrosis stage in alcoholic liver disease (ALD) [2]. Discontinuation of alcohol consumption makes steatosis disappear relatively rapidly (in weeks), and after longer periods even fibrosis processes are reduced [2].

This study aimed to investigate by optical microscopy the changes occurring in the liver parenchyma following ethyl alcohol administration for 70 days to Wistar rats, as well as the effect of administration of Tuşnad mineral water.

\section{Material and method}

For this study, 30 white male Wistar rats with a mean weight of 350 grams were used.

Materials: ethyl alcohol 12\%, mixed carbonated mineral water from spring 3 in Băile Tuşnad, tap water.

The natural mineral water from spring 3 in Băile Tuşnad has a total mineral content of 
$3351.0 \mathrm{mg} / \mathrm{l}$, according to data provided by the spa treatment facility. According to the laboratory test report, this is a mixed carbonated mineral water (CO2 $1646.0 \mathrm{mg} / \mathrm{l}$ ), with sodium chloride content (chlorine ions $617 \mathrm{mg} / \mathrm{l})$. Its composition also includes HCO3 $378.2 \mathrm{mg} / \mathrm{l}$, sodium $288 \mathrm{mg} / \mathrm{l}$, calcium $93.8 \mathrm{mg} / \mathrm{l}$, potassium $76 \mathrm{mg} / \mathrm{l}$, magnesium 35.5 $\mathrm{mg} / \mathrm{l}$, iron $17.3 \mathrm{mg} / \mathrm{l}$ and bromine traces. It is weakly iodinated and acidulated, it has a $\mathrm{pH}$ of 6 , and a temperature of $14.5^{\circ} \mathrm{C}$.

This study was performed with the approval of the Ethics Committee of the University of Medicine and Pharmacy ClujNapoca, number 533/23.12.2015, and the approval of the Sanitary Veterinary and Food Safety Authority Cluj, number 17/ 13.12.2016.

The animals were divided into two groups, as follows: the control group (M) -5 animals, and the experimental group (E) - 25 animals. The animals were accommodated at the biobase of the "Iuliu Hațieganu" UMF Cluj-Napoca, they were fed granular food, and animals in group $\mathrm{M}$ drank tap water, while animals in group $\mathrm{E}$ drank water with ethyl alcohol $12 \%$ for 70 days, 12-15 ml alcohol/animal/day. After this period, 2 animals in group $\mathrm{M}$ and 5 animals in group $\mathrm{E}$ were euthanized, and liver samples were collected for microscopic investigations. The rest of the animals were transferred to the Băile Tușnad spa for 50 days, where they benefited from similar maintenance conditions and received the same food. Group E animals were divided into two equal subgroups: group E1 (10 animals), which drank tap water, while group E2 (10 animals) drank mineral water from spring 3 in Băile Tuşnad, 50-75 $\mathrm{ml} /$ day/animal. At the end of the experiment, the animals were euthanized and liver fragments were collected, which were histologically processed. The liver fragments were harvested in the form of $3 \mathrm{~mm}$ thick slices. These were fixed by immersion in Stieve solution for 20 hours, after which they were post-fixed in $10 \%$ formalin for 72 hours. At the end of fixation, they were histologically processed by paraffin embedding, and $4 \mu \mathrm{m}$ thick sections were made. The sections were displayed on slides and were stained using
Masson's trichrome method modified by Goldner.

\section{Results and discussions}

In the liver sections examined at the end of the ethyl alcohol administration period (group E), it was observed that the general cytoarchitecture of the liver was similar to that of the control group (Fig. 1). No necrosis or the presence of inflammatory infiltrate was seen on the section surface. The cytoplasmic appearance of hepatocytes was slightly different compared to that of the control group, with typical hepatic steatosis aspects consisting of small cytoplasm gaps of quasiround shape, with different diameters.

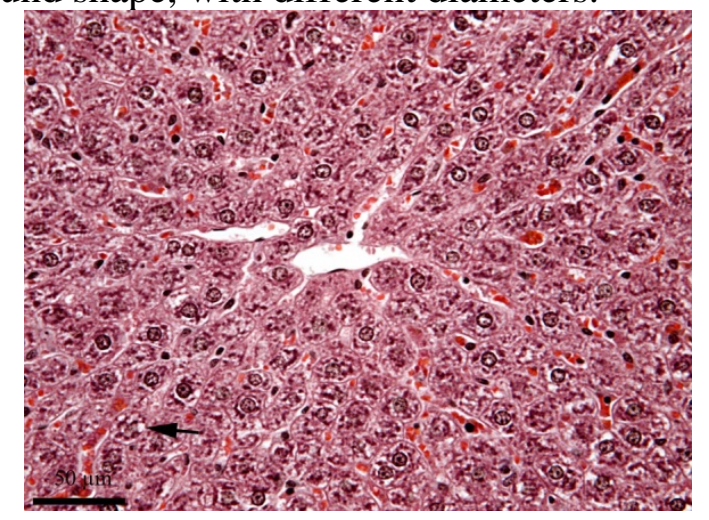

Fig. 1 Liver in group $\mathrm{M}$ - centrilobular area; black arrow - rare hepatocytes with lipid inclusions; Goldner's trichrome stain

In the majority of the hepatocytes, hepatic steatosis aspects with small droplets were present, and only in some lobules, particularly at their periphery, some hepatocytes showed large droplet steatosis (Fig. 2); rare cells with peripheral dislocation of the nucleus could also be seen.

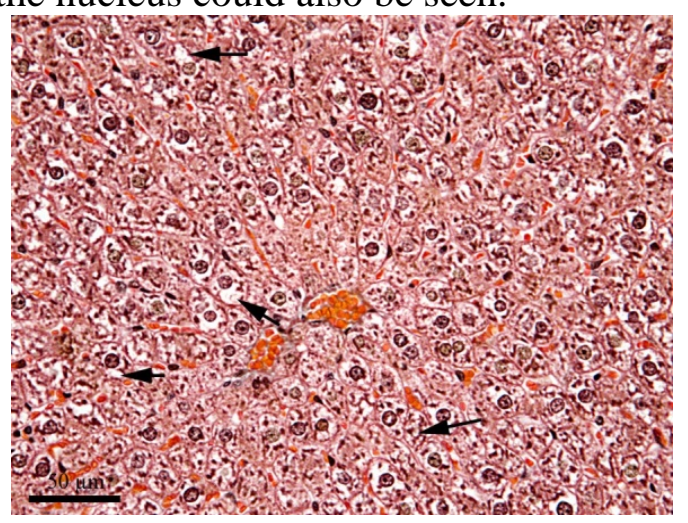

Fig. 2 Liver in group E - centrilobular area; black arrow - hepatic steatosis; Goldner's trichrome stain 
In lobules with pronounced hepatic steatosis processes, these were accompanied by mild fibrosis in the centrilobular venule. In lobules, hepatocytes showed large droplet steatosis, but fibrosis processes were also seen in the perilobular venule (Fig. 3). On the examined section surface, no venous shunts between the perilobular and the centrilobular venule were observed.

The fibrosis process in the centrilobular venule as well as in the perilobular venule was not very advanced, because venules were not surrounded by thick collagen fibers. Young connective tissue proliferated around the vessels, in which most of the collagen fibers were thin. The small number of fibroblasts present in the connective tissue proliferated around the vessels showed the fact that fibrosis was not very advanced. The presence of fibroblasts also suggested that this fibrosis process was not completed.

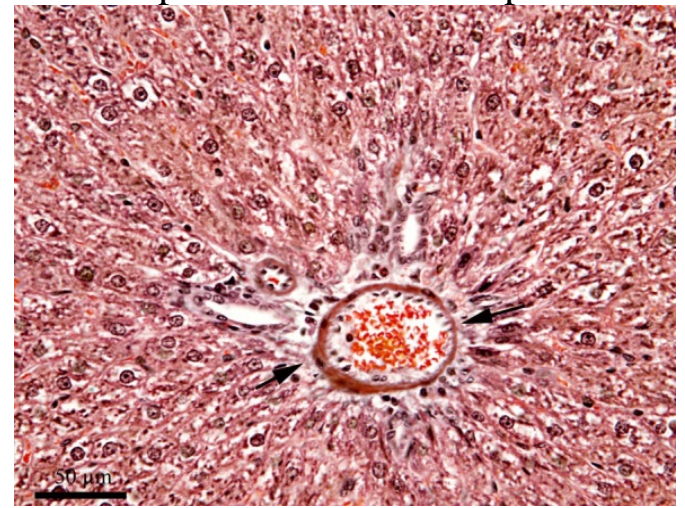

Fig. 3 Liver in group $\mathrm{E}$ - the portobiliary space; black arrow - perivascular fibrosis;

Goldner's trichrome stain

At the end of the period during which the animals drank tap water (group E1) and mineral water (group E2), respectively, the general aspects were similar to those previously described.

The pathological findings in group E1 animals compared to those described after completion of ethyl alcohol administration seemed to be slightly more pronounced, even if ethyl alcohol was no longer administered. More hepatocytes were affected by steatosis and at the same time, lipid inclusions seemed to be larger in diameter (Fig. 4). Also, fibrosis processes in both the centrilobular and perilobular venules were more advanced, with a more consolidated appearance of the connective tissue (Fig. 5). The degree of lipid loading of hepatocytes in group E2 rats was higher than in rats euthanized after completion of alcohol consumption, and lower compared to group E1 (Fig. 6).

The fibrosis process around the centrilobular and perilobular venules in group E2 rats was more consolidated compared to rats euthanized at the end of the ethyl alcohol administration period. There were no significant differences between groups E1 and E2 regarding venule fibrosis.

The pathological findings in the liver of rats in groups E1 and E2 were more advanced than those present at the end of the ethyl alcohol administration period (group E). These results are in contradiction to those reported by Theise (2013), who maintains that in humans, hepatic steatosis disappears in several weeks.

The results obtained in this study show that 50 days after discontinuation of ethyl alcohol administration in rats, steatosis was more pronounced compared to the day when alcohol administration was discontinued.

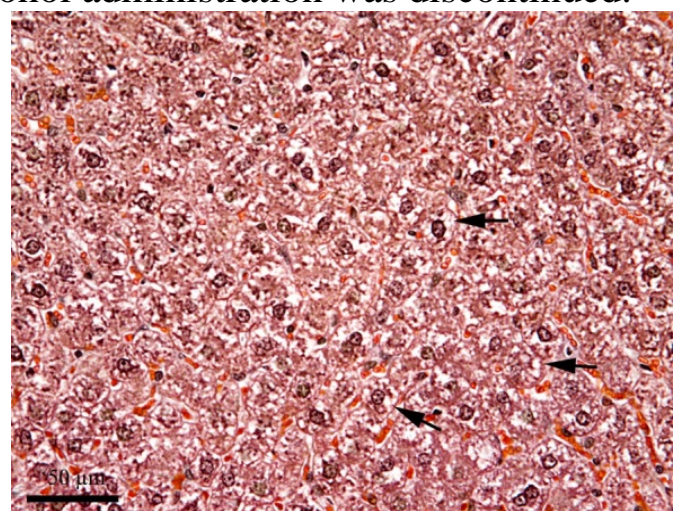

Fig. 4 Liver in group E1 - appearance of hepatocytes; black arrow - steatosis; Goldner's

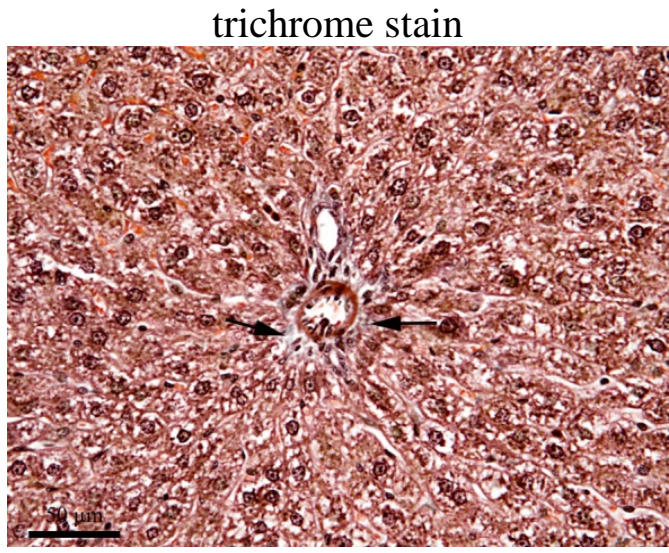


Fig. 5 Liver in group E1 - portobiliary space; black arrow - perivascular fibrosis; Goldner's trichrome stain

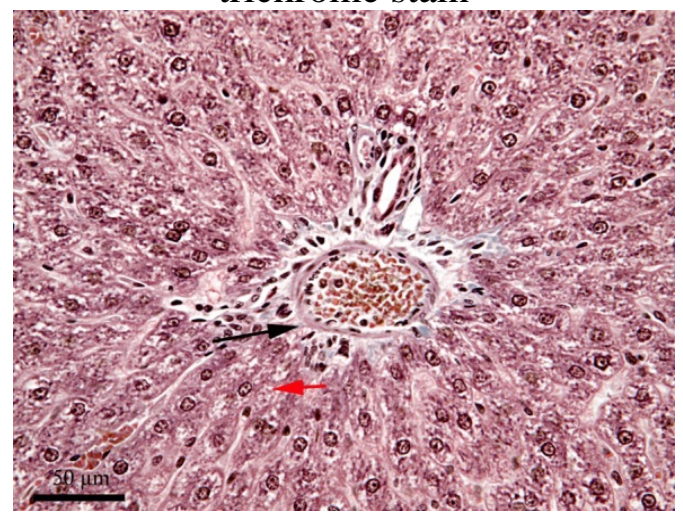

Fig. 6 Liver in group E2 - portobiliary space black arrow - perivascular fibrosis; red arrow

- steatosis; Goldner's trichrome stain

These differences seem to be due to the

fact that lesions detectable by optical microscopy investigations do not develop very rapidly, but they appear after a longer time period.

Our findings in the liver of rats are confirmed by other authors $[1,6]$. Regarding the degree of hepatocyte involvement, this is not uniform. Thus, there are lobules in which only a few hepatocytes in the inner third of the lobules are affected, as well as lobules in which lesions extend to hepatocytes in the outer third of the lobules. In these lobules with affected hepatocytes in the outer third, the degree of lipid loading is higher. These aspects described by us are supported by another author [2], according to whom lesions are more severe as they extend to the periphery of the lobule. We obtained similar results in a previous study, in which the first stage of the experiment was identical to that of this study, but in the second stage, the period during which the animals were administered mineral water was 20 days shorter, i.e. only 30 days. The results found after the first 70 days in the two experimental studies are similar, but the findings at the end of the experiments are different: in the second study, the degree of fat loading of hepatocytes in group E2 animals is lower than described by us in a previous study [7].

Perivascular fibrosis processes described by us were also reported by other authors $[2,4,6]$, but we found no necrotic and inflammatory aspects like those described by Lieber [4].

The differences between groups E1 and $\mathrm{E} 2$ regarding the degree of fat loading of hepatocytes can be attributed to the curative effect of the mixed carbonated mineral water from spring 3 in Băile Tuşnad compared to the toxic action of ethyl alcohol.

\section{Conclusions}

Following ethyl alcohol administration for 70 days to rats, hepatic steatosis and mild perivascular fibrosis aspects were observed in the liver.

The degree of fat loading of hepatocytes and perivascular fibrosis aspects on day 120 in group E1 rats were more advanced compared to those present on day 70 in group E rats.

Mineral water administration for 50 days to rats (group E2) that previously consumed ethyl alcohol for 70 days led to a reduction in the degree of fat loading of hepatocytes compared to the hepatocytes of rats that drank tap water (group E1).

\section{Bibliography}

1. Louvert A., Mathurin P. Alcoholic liver disease: mechanisms of injury and targeted treatment. Nature Reviews Gastroenterology \& Hepatology, 12: 231-242, 2015.

2. Theise N.D. Histopathology of Alcoholic Liver Disease. Clinical Liver Disease, 2(2): 64-67, 2013. 3. Maher J.J. Alcoholic steatosis and steatohepatitis. Semin Gastrointest Dis., 13(1):319, 2002.

4. Lieber C.S. Alcoholic fatty liver: its pathogenesis and mechanism of progression to inflammation and fibrosis. Alcohol, 34(1):9-19, 2004.

5. Parés A., Caballería J., Bruguera M., Torres M., Rodés J. Histological course of alcoholic hepatitis. Influence of abstinence, sex and extent of hepatic damage. J Hepatol., 2(1):33-42, 1986.

6. Tsukamoto H., Towner S.J., Clofalo L.M., French S.W. Ethanol-induced liver fibrosis in rats fed high fat diet. Hepatology, 6(5): 814-822, 1986. 7. Dogaru G., Motricală M., Molnár Á., Rus V. An experimental study regarding the biological effects of mineral water from spring 3 in Băile Tuşnad on some organs after ethyl alcohol administration. Balneo Research Journal, 7(1), 2328, 2016. 